\title{
The Effect of Aging on Inhibition in Task Switching: Controlling for Episodic Retrieval
}

\author{
James A. Grange and Agnieszka W. Kowalczyk \\ School of Psychology, Keele University, UK
Draft version as of October 2, 2017. This paper has not been peer reviewed. Please do not copy or cite without author's permission.

\begin{abstract}
Inhibition in task switching is inferred from n-2 task repetition costs: responses are slower and less accurate to ABA task switching sequences than CBA sequences, thought to reflect the persisting inhibition of task $\mathrm{A}$ in an ABA sequence which hampers re-activation attempts. Despite the inhibitory deficit hypothesis of age-related decline in cognition, studies have found no consistent age-related difference in n-2 task repetition costs. Recent work has shown that extant measures of the n-2 task repetition cost are contaminated with episodic retrieval effects, which inflate estimates of inhibition. The current study revisited potential age-related differences in $\mathrm{n}-2$ task repetition costs in a design that controls for episodic retrieval. We find equivalent $n-2$ task repetition costs in older and younger adults in response times, which provides converging evidence of no age-related decline of inhibition in task switching.
\end{abstract}

Keywords: Task switching; inhibition; episodic retrieval; aging

Cognitive inhibition can be defined as "...the stopping or overriding of a mental process, in whole or in part, with or without intention" (p.5 MacLeod, 2007). Inhibitory control has been suggested to play a role in many cognitive operations (e.g., Gorfein \& MacLeod, 2007), and is thought to facilitate the selection of goal-relevant information by suppressing goal-irrelevant information.

Cognitive inhibition is of interest to cognitive gerontologists because an important theory of cognitive decline due to healthy aging is the inhibition deficit hypothesis of Hasher and colleagues (e.g., Hasher, Lustig, \& Zacks, 2007; Hasher \& Zacks, 1988; Hasher, Zacks, \& May, 1999), which suggests that inhibition becomes less efficient as we age. Despite this hypothesis, the evidence for age-related decline in inhibition is mixed. For example,

Please address correspondence to James A. Grange, School of Psychology, Dorothy Hodgkin Building, Keele University, Keele, UK, ST5 5BG. Email: grange.jim@gmail.com. All raw data, analysis code, and code to run the experiment can be downloaded from https://osf.io/wvncy/. We are grateful to Chloe Waterman and Dominic Bromley for collecting the data. 
Kramer, Humphrey, Larish, and Strayer (1995) found negative age-related effects on stopsignal performance and the Wisconsin Card Sorting Task, but no age-related effects on other inhibition measures, such as negative priming, the response-compatibility effect, and every-day cognitive failures. In meta-analytical studies, it has been shown that there is no consistent age-related effects on inhibition as measured by the negative priming task (Gamboz, Russo, \& Fox, 2002), inhibition of return (Verhaeghen, 2011), or the Stroop task (Verhaeghen \& De Meersman, 1998).

These inconsistent age-related effects on inhibition measures could be explained by inhibition being a multi-faceted process (Friedman \& Miyake, 2004; Nigg, 2000), with agerelated decline on only some aspects (Kramer et al., 1995). However, the inconsistency in findings could be also due to the experimental measures not actually capturing inhibitory control. Within cognitive psychology, inhibition as a construct is controversial because many behavioral phenomena suggested to reflect an active inhibitory mechanism can often be explained by non-inhibitory accounts (Gorfein \& Brown, 2007; MacLeod, Dodd, Sheard, Wilson, \& Bibi, 2003). If we wish to understand whether cognitive decline in healthy aging is driven by an inhibitory deficit, it is important that we examine closely whether our behavioral paradigms are indeed measuring inhibition.

There appears to be compelling evidence for a role of inhibition in task switching designs, which is the focus of the current paper. In the next section, a brief overview of inhibition in task switching is provided, together with an overview of studies which have examined potential age-related differences in this domain. Then, an overview of a noninhibitory account of inhibition effects in task switching (episodic retrieval) is provided; episodic retrieval has recently been shown to confound measures of task inhibition (Grange, Kowalczyk, \& O'Loughlin, 2017). We then present an experiment which controls for this confound and assesses measures of inhibition in older and younger adults.

\section{Inhibition in Task Switching}

Task switching designs present participants with simple cognitive tasks (such as making parity or magnitude judgments on number stimuli), and they are required to switch between them; participants are informed on a trial-by-trial basis what the current task is by way of a cue (e.g., the word "magnitude"). The task switching paradigm has been utilized to probe many aspects of cognitive control (see Grange \& Houghton, 2014; Kiesel et al., 2010; Vandierendonck, Liefooghe, \& Verbruggen, 2010, for reviews), and has also been used to assess cognitive control in healthy aging (see Grange \& Becker, in press; Wasylyshyn, Verhaeghen, \& Sliwinski, 2011, for meta-analyses of findings).

Behavioral evidence for a possible role of inhibition in task switching comes from the so-called $n-2$ task repetition cost: When switching between three possible tasks (arbitrarily labelled A, B, and C), response times (RTs) and errors are increased on ABA task sequences (n-2 task repetitions) than CBA sequences ( $\mathrm{n}-2$ task switches; Mayr \& Keele, 2000). The $\mathrm{n}-2$ task repetition cost is thought to reflect the inhibition of task A which is needed to facilitate the switch to task B (from A-B); this inhibition of task A persists for a short while, and thus hinders re-activation attempts if this task is required soon after it has been inhibited (as is the case in an ABA sequence). The $\mathrm{n}-2$ task repetition cost is important to research on cognitive inhibition more widely because it is thought to be resistant to non-inhibitory explanations (Koch, Gade, Schuch, \& Philipp, 2010). 
As a consequence, the $\mathrm{n}-2$ task repetition cost appears to be an ideal measure to assess potential inhibition deficits in healthy aging. Studies to date, though, have presented an unclear picture of age-related differences in this cost. The first study to examine this was Mayr (2001, Experiment 1), who found larger n-2 task repetition costs in older adults in comparison to younger adults. This increase in $\mathrm{n}-2$ task repetition cost in older adults was also found by Pettigrew and Martin (2016). However, Lawo, Philipp, Schuch, and Koch (2012) found statistically-equivalent $\mathrm{n}-2$ task repetition costs in older and younger adults, a pattern of results also found by Schuch (2016) and Rey-Mermet, Gade, and Oberauer (in press). However, note that Schuch (2016) found some evidence for impairments in older adults at the latent level when modelling the data using the diffusion model of choice RT. Thus, despite the inhibition deficit hypothesis of Hasher and colleagues (Hasher et al., 2007, 1999), no consistent age-related difference has been found for the $\mathrm{n}-2$ task repetition cost. If anything, when a difference is present, $n-2$ task repetition costs are larger in older adults than younger (Mayr, 2001; Pettigrew \& Martin, 2016).

\section{A Key Non-Inhibitory Account: Episodic Retrieval}

One key non-inhibitory account of the $\mathrm{n}-2$ task repetition cost is that of episodic retrieval. On a single task switching trial, participants are (typically) presented with a visual cue, a visual target, and on the basis of these perceptual elements a response must be selected and executed. For example, consider Figure 1, which demonstrates the experimental paradigm used in the current paper (based on the paradigm introduced by Mayr, 2002). In this task, participants are presented with a circular target; participants are required to mentally make a spatial transformation of this target according to one of three rules (either a "vertical", "diagonal", or a "horizontal" transformation); participants then must make a spatially-congruent response to the location where the target would move to given the current rule. For example, if the target was presented in the top-right and the current rule was a horizontal transformation, the correct response would be a "top-left" key press. Participants know which rule is relevant on the current trial by way of a shape cue presented in the center of the screen (a hexagon for the vertical judgement; a square for the diagonal judgement; and a triangle for the horizontal judgement).

According to the episodic retrieval account, when a trial has been performed, an episodic trace of all parameters of the trial is stored in memory; according to the theory of event coding (Hommel, 2004), such an episodic trace might be a representation consisting of the bound information of the cue presented, the target presented, and the response that was executed. When this task is cued again, this episodic trace is automatically retrieved (e.g., Logan, 1988). If the parameters of the current trial differ to that contained in the retrieved episodic trace (e.g., a "top-left" response was coded in the episodic trace, but the current trial requires a "bottom-right" response), a mismatch $\operatorname{cost}^{1}$ occurs relative to if the

\footnotetext{
${ }^{1}$ Note that in the event file literature, this scenario would be referred to as a "partial repetition" of features (e.g., Spapé \& Hommel, 2014), because although the stimulus is in a different location-and hence a difference response is required - to that of the retrieved episodic trace, the cue remains the same across the ABA sequence. Partial repetitions such as these reliably produce costs to performance in contrast to complete mismatches where all parameters differ to the retrieved trace, which actually produce benefits to performance. The current study does not present any complete mismatches, so the term "mismatch" in the current paper refers to partial repetitions. We use the former term for simplicity.
} 


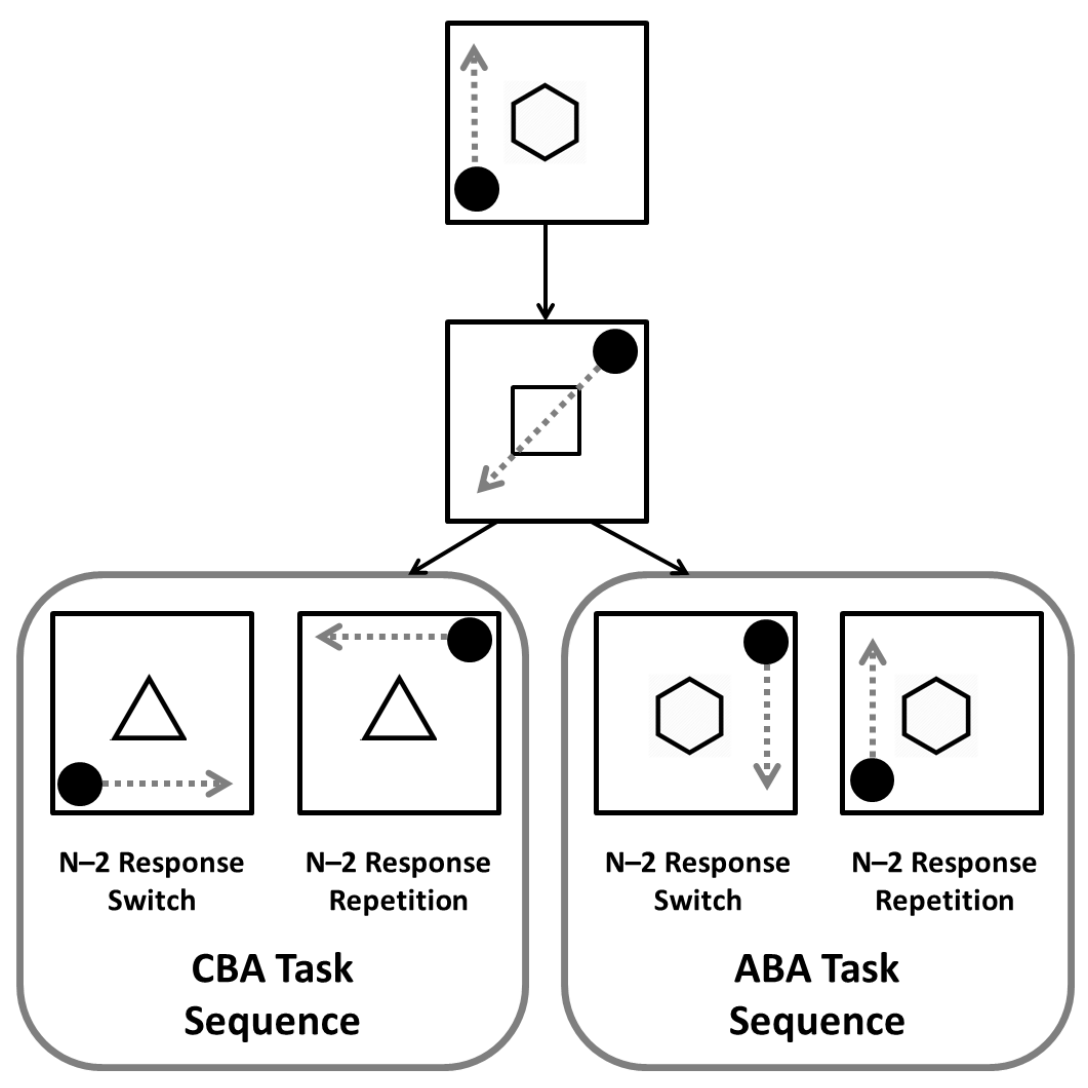

Figure 1. Overview of the experimental paradigm used in the current experiment. Dotted arrows represent the spatial transformation that was required on each trial, but was not shown to the participant. Time runs from top to bottom. Images not to scale. Figure available at https://www.flickr.com/photos/150716232@N04/shares/5413G0 under CC licence https://creativecommons.org/licenses/by/2.0/.

parameters match (which facilitates performance because the correct response is primed by the retrieved episodic trace). Such an account can potentially explain the $\mathrm{n}-2$ task repetition cost as a mismatch cost rather than due to inhibition, because the current trial's parameters will typically differ for task A across an ABA sequence.

Due to the simplicity of the stimulus display in the above-mentioned paradigm, it is possible to control whether the trial parameters match or mismatch across an ABA sequence (see right side of Figure 1): If the target is presented in the same location across an ABA sequence, the required response will also be the same (i.e., an n-2 response repetition); if, however, the target is in a different location, the response will be different (i.e., an n-2 response switch). Note that $n-2$ response repetitions and switches are controlled in CBA sequences, too.

The episodic retrieval account predicts an n-2 task repetition cost only for n-2 re- 
sponse switches, because the trial parameters will not match those of the retrieved episodic trace; for $\mathrm{n}-2$ response repetitions, because there is a direct match between current trial parameters and the retrieved episodic trace, the episodic retrieval account predicts an $n-2$ task repetition benefit, because the match will prime performance. An inhibition account would predict an equivalent $\mathrm{n}-2$ task repetition cost for $\mathrm{n}-2$ response repetitions and switches, because in both cases inhibition of task A will persist across the ABA sequence.

These contrasting predictions were tested by Mayr (2002), who found statisticallyequivalent $\mathrm{n}-2$ task repetition costs for $\mathrm{n}-2$ response repetitions and $\mathrm{n}-2$ response switches. That there was an $\mathrm{n}-2$ task repetition cost for $\mathrm{n}-2$ response repetitions - where the episodic parameters perfectly match the current trial's parameters-presented evidence against the episodic retrieval account of the $\mathrm{n}-2$ task repetition cost.

This topic was revisited by Grange et al. (2017) who were interested in whether there was any modulatory effect of episodic retrieval on the $\mathrm{n}-2$ task repetition cost. Across three experiments, they found evidence of reduced $n-2$ task repetition costs when episodic retrieval was controlled (i.e., for $\mathrm{n}-2$ response repetitions). On the basis of these findings, Grange and colleagues suggested that the $\mathrm{n}-2$ task repetition cost - as typically measuredis a confounded mixture of inhibition plus episodic retrieval.

\section{The Current Study}

The purpose of the current study was to revisit the effect of healthy aging on inhibition in task switching as measured by the $\mathrm{n}-2$ task repetition cost, but by controlling for episodic retrieval using the paradigm presented by Mayr (2002) and extended by Grange et al. (2017). Previous work which has shown inconsistent effects of age on the n-2 task repetition cost did not control for episodic retrieval, so the conclusion to be drawn from such mixed findings is difficult to establish. It could be that there is a consistent age-related effect on inhibition which is obscured by episodic retrieval. If this is the case, we would expect smaller $n-2$ task repetition costs for older adults under conditions of episodic matches (i.e., n-2 response repetitions). In contrast, finding no age-related effect on the $\mathrm{n}-2$ task repetition cost when controlling for episodic retrieval would strengthen the conclusion that there is no age-related effect on inhibition. The current study therefore has important theoretical implications for theories of cognitive inhibition related to aging.

\section{Method}

\section{Participants}

This study received ethical approval from Keele University's Ethics Review Panel. Thirty-one older adults (age range 65-80) and 31 younger adults (age range 18-25) were recruited for the current study. Two older adults and two younger adults were removed from the analysis for session-wise accuracy below the pre-specified criterion of $90 \%$. Older adults were healthy, community-dwelling individuals recruited from the local area; they self-declared no clinical diagnosis of dementia or depression. Younger adults were recruited from Keele University. Older adults were provided $£ 20$ as compensation for their time, and younger adults were provided $£ 10$ (older adults attended a 2-hour session, younger adults a 1-hour session). All participants had normal or corrected-to-normal vision. 
Older adults were screened for cognitive impairment using the Mini-Cog assessment (Borson, Scanlan, Brush, Vitaliano, \& Dokmak, 2000), with possible scores ranging from $0-5$. A score below 3 on this test suggests cognitive impairment. No participant met this criterion $(\mathrm{M}=4.6, \mathrm{SD}=0.56)$. Older adults were also screened for depression using the Geriatric Depression Scale-Full Version (Yesavage, Brink, \& Rose, 1983), with possible transformed-scores ranging from 0-30. A score from 0-9 is classified as "normal", 10-19 is suggesting of "mild depression", and 20-30 as "severe depression". All participants scored below 10 , with the exception of one participant who scored 10 (overall $\mathrm{M}=2.93, \mathrm{SD}=$ 2.79). No older adults were removed on the basis of these scores.

\section{Apparatus \& Stimuli}

Stimuli were presented on a 17in. monitor connected to a PC. The experiment was programmed and presented using PsychoPy (Pierce, 2007). Responses were collected via a $1 \mathrm{~ms}$-precise USB keyboard. The stimulus display consisted of a $7 \times 7 \mathrm{~cm}$ black square frame on a grey background. The target was a black circle measuring $1.2 \mathrm{~cm}$ in diameter. Possible cues were polygon shapes: a hexagon, a square, or a triangle; all were approximately $3 \mathrm{~cm}$ in height and width, and had a black outline (with no fill).

\section{Procedure}

The task required the participant to make a spatially-compatible response to where a circular target presented in one of the four corners of the square frame would move to according to the currently-relevant spatial transformation rule. Participants knew which rule was relevant on the current trial by way of a task cue.

Each trial began with the presentation of a blank square frame for $150 \mathrm{~ms}$. After this time, a cue was presented in the centre of the frame for $150 \mathrm{~ms}$, after which the circle target appeared in one of the four corners. The cue remained in the centre of the frame during target presentation, and both remained visible until the participant made a response. Participants were instructed to use the cue to retrieve from memory which spatial transformation was relevant on the current trial. If the cue was a hexagon, they had to make a vertical transformation; if the cue was a square, they had to make a diagonal transformation; and if the cue was a triangle, they had to make a horizontal transformation. Responses were made on a numerical keypad using the keys "1", "2", "4", and "5" to represent a "bottom-left", "bottom-right", "top-left", and "top-right" response, respectively. Figure 1 demonstrates sample responses for each transformation. Instructions asked participants to respond as quickly and as accurately as possible. Participants were required to use their right-index finger for their response, and to reset their finger location to the center of the four response keys after their responses. Once the participants made a response, the cue and target were removed from the square frame and the next trial began. If participants made an error, the word "Error!" appeared in the center of the screen in red font for 1,000ms.

The cue for each trial was selected randomly with the constraint that no immediate task repetitions could occur, as inclusion of immediate task repetitions has been shown to reduce the magnitude of the $\mathrm{n}-2$ task repetition cost (Philipp \& Koch, 2006). Target location was selected randomly on each trial. Hence, the probability of a response repetition from $\mathrm{n}-2$ to $\mathrm{n}$ is .25 . The experimental section consisted of 8 blocks with 60 trials in each 
block; a self-paced rest screen followed each block. The experimental section was preceded by a practice section consisting of one block of 16 trials. If participants made four or more errors during this practice, the practice was repeated once. After this, the experimental section was initiated.

\section{Design}

The experiment had three factors in a mixed design: Age (older vs. younger), Task Sequence (ABA sequence vs. CBA sequence) and Response ( $\mathrm{n}-2$ response repetition vs. n-2 response switch). Age was a between-subjects variable, and Task Sequence and Response were manipulated within-subjects. The dependent variables were response time (RT) measured in milliseconds (ms) and proportion accuracy.

\section{Results}

\section{Analytical Approach}

Analysis was conducted on mean RT in milliseconds and accuracy measured as proportion scores. To complement the mean RT analysis we also analysed log-transformed RTs - specified as $\log (\mathrm{RT})$ - to compensate for the typical large baseline differences in mean RT between older and younger adults. To facilitate report of the results, $\log (\mathrm{RT})$ analysis is only reported for interactions involving the factor of Age. We also complement traditional null-hypothesis significance testing with Bayesian analysis for factorial designs (Rouder, Morey, Verhagen, Swagman, \& Wagenmakers, 2017). This approach is discussed in more detail in the relevant sections. Analysis was conducted using R Statistics (R Core Team, 2015); the Bayesian analysis was conducted using the BayesFactor package for R (Morey \& Rouder, 2015).

For all analyses, the first two trials of each block were removed as they cannot be classified as ABA or CBA sequences. For the RT analysis, error trials and the two trials following an error were also removed. In addition, RTs shorter than $150 \mathrm{~ms}$ and longer than 2.5 standard deviations above the mean for each participant for each cell of the experimental design were also removed. Final RTs and proportion accuracy are shown in Table 1.

\section{Response Time}

There was a main effect of Age, with the expected longer RTs for older adults $(\mathrm{M}=$ $1667, \mathrm{SE}=36)$ compared to younger adults $(\mathrm{M}=1069, \mathrm{SE}=28), F(1,56)=45.16, p<$ $.001, \eta_{G}^{2}=.43$. There was also a main effect of Task Sequence, with longer RTs to ABA sequences $(\mathrm{M}=1406, \mathrm{SE}=43)$ than to $\mathrm{CBA}$ sequences $(\mathrm{M}=1329, \mathrm{SE}=42), F(1,56)=$ 44.21, $p<.001, \eta_{G}^{2}=.01$. There was a main effect of Response, with shorter RTs to $\mathrm{n}-2$ response repetitions $(\mathrm{M}=1347, \mathrm{SE}=42)$ than to $\mathrm{n}-2$ response switches $(\mathrm{M}=1389, \mathrm{SE}=$ 43), $F(1,56)=9.70, p<.01, \eta_{G}^{2}=.004$.

There was no interaction between Age and Task Sequence, $F(1,56)=0.14, p=.71$, $\eta_{G}^{2}<.001$. This interaction remained non-significant in the $\log (\mathrm{RT})$ analysis, $F(1,56)=$ $1.05, p=.31, \eta_{G}^{2}<.01$. The $\mathrm{n}-2$ task repetition cost was $82 \mathrm{~ms}$ for the older adults and $72 \mathrm{~ms}$ for the younger adults. There was also no interaction between Age and Response, 
$F(1,56)=0.76, p=.39, \eta_{G}^{2}<.001$. For older adults, RTs for $\mathrm{n}-2$ response repetitions were $30 \mathrm{~ms}$ shorter than for $\mathrm{n}-2$ response switches; for younger adults, this difference was $54 \mathrm{~ms}$.

Replicating earlier work (Grange et al., 2017), there was a significant interaction between Task Sequence and Response, $F(1,56)=17.12, p<.001, \eta_{G}^{2}=.004$. N-2 task repetition costs were $36 \mathrm{~ms}$ ( $\mathrm{SE}=19)$ for $\mathrm{n}-2$ response repetitions, compared to $118 \mathrm{~ms}$ ( $\mathrm{SE}$ $=11$ ) for $\mathrm{n}-2$ response switches. Importantly for the current work, the three-way interaction between Age, Task Sequence, and Response was not significant, $F(1,56)=0.05, p=.82$, $\eta_{G}^{2}<.001$. This interaction remained non-significant in the $\log (\mathrm{RT})$ analysis, $F(1,56)=$ $0.95, p=.33, \eta_{G}^{2}<.001 .^{2}$

Table 1

Mean response time (RT) in milliseconds (ms) and proportion accuracy data as a function of task sequence (ABA vs. CBA), age group (older vs. younger adults) and $n$-2 response (repetition vs. switch). Standard errors shown in parentheses.

\begin{tabular}{ccccccc}
\hline & & \multicolumn{3}{c}{ Task Sequence } \\
\cline { 3 - 4 } Age Group & n-2 Response & RT (ms) & Accuracy & & \multicolumn{2}{c}{ CBA (ms) } \\
\hline \multirow{2}{*}{ Older Adults } & Repetition & $1673(74)$ & $0.972(0.006)$ & & $1631(72)$ & $0.978(0.005)$ \\
& Switch & $1742(71)$ & $0.975(0.004)$ & & $1622(71)$ & $0.980(0.004)$ \\
Younger Adults & & & & & \\
& Repetition & $1057(52)$ & $0.969(0.006)$ & & $1027(50)$ & $0.956(0.008)$ \\
& Switch & $1153(64)$ & $0.948(0.006)$ & & $1038(57)$ & $0.973(0.004)$ \\
\hline
\end{tabular}

Bayesian Analysis. To complement the standard null-hypothesis significance testing (NHST) of the three-way interaction, we modelled the $\mathrm{n}-2$ task repetition cost data in Figure 2, which shows a two-way design with Response and Age as factors, and the n-2 task repetition cost as the dependent variable. Complementing the standard analysis with Bayesian analysis is important because a null-hypothesis cannot be accepted using NHST. As our main finding of the RT analysis is a null interaction, we wanted to be able to quantify support for this null, which can be achieved using Bayesian analysis.

The Bayesian analysis utilised the Bayesian model comparison technique for factorial designs as introduced by Rouder et al. (2017). Bayes factors (BFs) were calculated for four separate models: (1) a main effect of Response; (2) a main effect of Age; (3) main effects of Age and Response; and (4) main effects of Age and Response, plus their interaction. All models included "Subject" as a random-effects factor. The BFs calculated are a ratio of the model's evidence - given the data - against a denominator of a "null" model which contains just the random factor.

Of theoretical interest to the current study is whether the model with two main effects plus their interaction is best supported by the data compared to the other models.

\footnotetext{
${ }^{2} \mathrm{As} \mathrm{n}-2$ response repetitions occurred with probability $=.25$, it could be possible that this imbalance is influencing the results. To address this issue, we conducted a linear mixed effects model analysis using the lme4 package (Bates, Mächler, Bolker, \& Walker, 2015) in R using the trial-level RT data. Therefore, this model takes the imbalance into account. The model - in lme4 syntax — was defined as RT Sequence * Response ${ }^{*}$ Age + (Sequence|Participant $)+$ (Response|Participant). All of the interactions involving age remained non-significant (absolute $t$-values less than 0.828).
} 


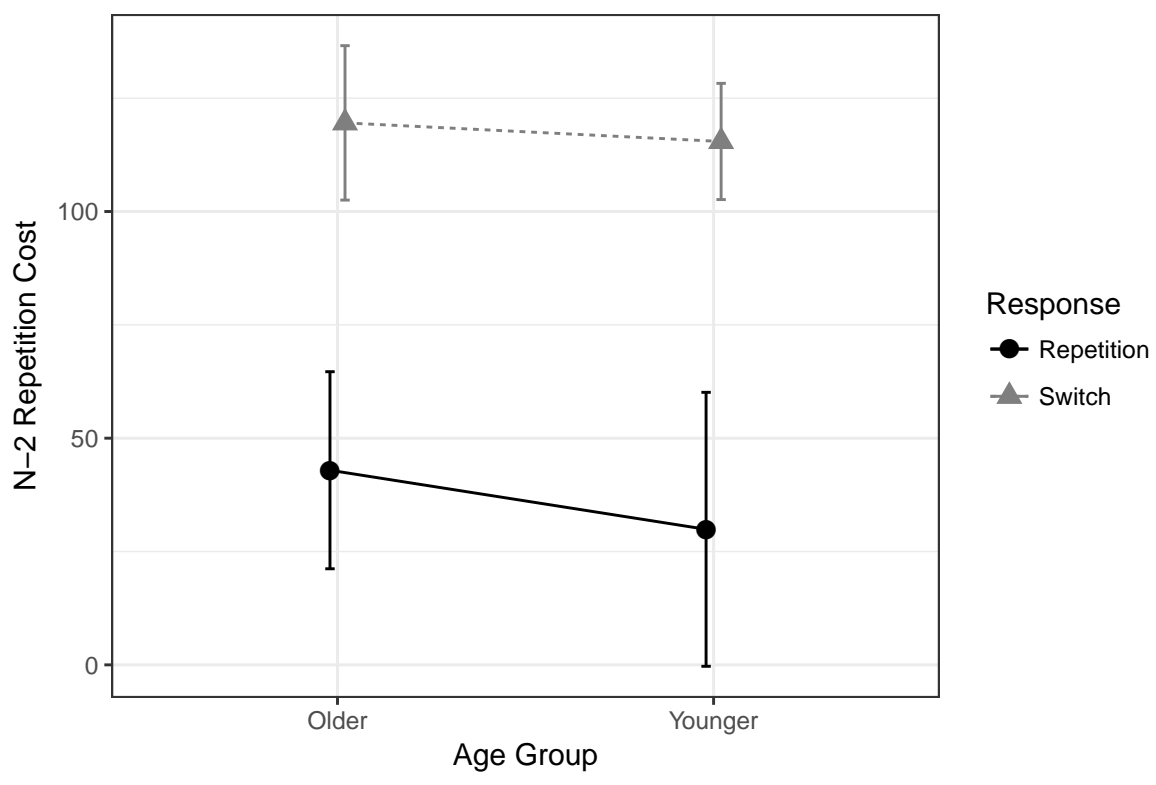

Figure 2. N-2 task repetition cost response time (RT) data in milliseconds (ms) for both levels of Response and Age. Error bars denote +/- 1 standard error around the mean.

Model comparison proceeded by finding the ratio of this model's BF compared to the best overvall model's BF (or the second-best model's BF if the two main effects plus interaction model was best overall). This comparison produces a new $\mathrm{BF}$ which is the ratio of evidence supporting the interaction model compared to the best (or next-best) model. BFs greater than one in this model comparison procedure indicate support for the interaction model and BFs less than one indicate support against the interaction model.

For the response time analysis (see upper panel of Table 2), the full main effects plus interaction model had a $\mathrm{BF}$ of 37.10; the overall best model was the model with a main effect of Response $(\mathrm{BF}=541)$. Thus, the $\mathrm{BF}$ for the critical model comparison is 37.10 $/ 541=0.069$. This suggests that the data are about 14 times more likely $(1 / 0.069)$ under the main effect of Response model, which provides "strong" evidence against the full interaction model.

For the $\log (\mathrm{RT})$ analysis (see middle panel of Table 2), the interaction model had a $\mathrm{BF}$ of 75.70 , and the overall best model was again the main effect of Response with a BF of 513. The BF for the critical model comparison is $75.70 / 513=0.148$. This suggests that the data are about 7 times more likely $(1 / 0.148)$ under the main effect of Response model, which provides "moderate" evidence against the full interaction model.

The Bayesian analysis supports the findings from the standard NHST outcome: $\mathrm{n}-2$ task repetition costs are larger for response switches than response repetitions, and this pattern is not different for older adults and younger adults. 


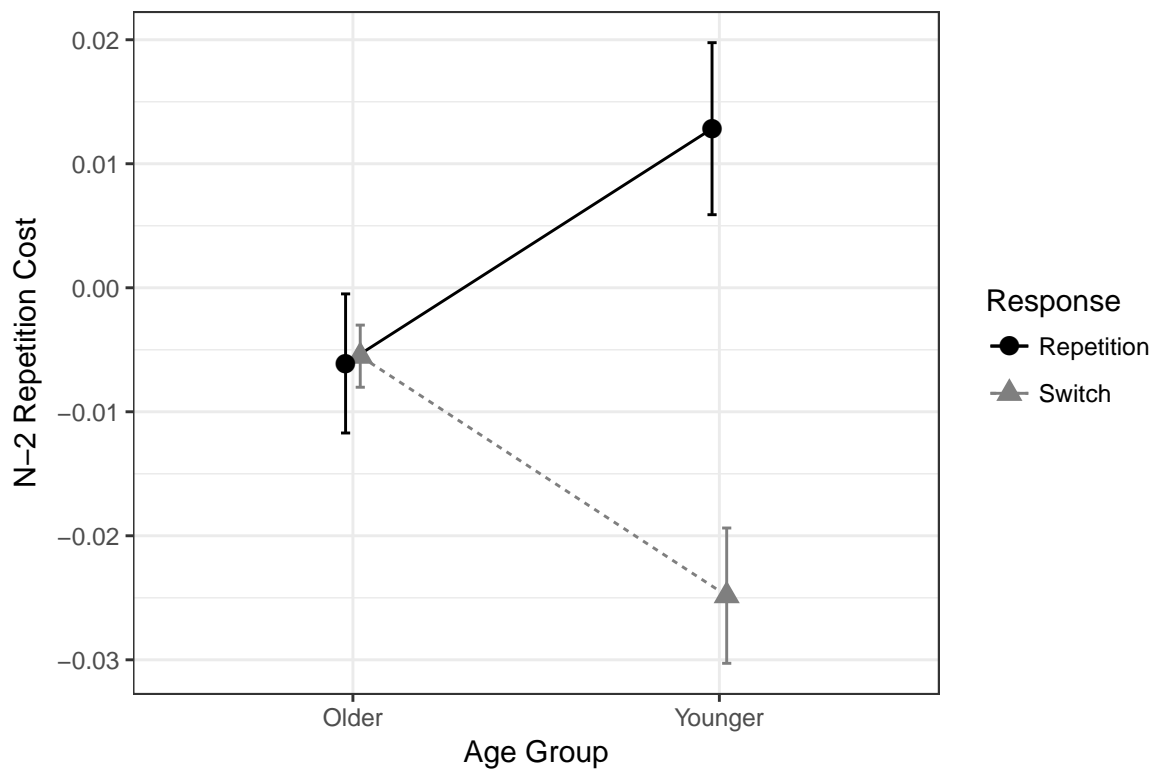

Figure 3. Proportion accuracy n-2 task repetition cost data for both levels of Response and Age. Error bars denote +/- 1 standard error around the mean.

\section{Accuracy}

For the accuracy data, there was a main effect of Age, with higher accuracy for older adults $(\mathrm{M}=0.976, \mathrm{SE}=0.002)$ than younger adults $(\mathrm{M}=0.962, \mathrm{SE}=0.003), F(1,56)=$ $5.44, p=.02, \eta_{G}^{2}=.06$. There was also a main effect of Task Sequence, with lower accuracy for $\mathrm{ABA}$ sequences $(\mathrm{M}=0.966, \mathrm{SE}=0.003)$ than for $\mathrm{CBA}$ sequences $(\mathrm{M}=0.972, \mathrm{SE}=$ $0.003), F(1,56)=5.23, p=.03, \eta_{G}^{2}=.01$. The main effect of Response was not significant, $F(1,56)<0.001, p=.98, \eta_{G}^{2}<.001$.

There was no interaction between Age and Task Sequence, $F(1,56)<0.01, p=.96$, $\eta_{G}^{2}<.001$ : The $\mathrm{n}-2$ task repetition cost was -0.006 for both older adults and younger adults. There was also no interaction between Age and Response, $F(1,56)=0.72, p=.40, \eta_{G}^{2}$ $=.001$. For older adults, responses for $\mathrm{n}-2$ response repetitions were 0.002 more accurate than for $\mathrm{n}-2$ response switches; for younger adults, this difference was -0.003 .

The interaction between Task Sequence and Response was significant, $F(1,56)=$ $11.06, p=.002, \eta_{G}^{2}=.02$. This interaction was further qualified by a significant three-way interaction between Task Sequence, Response, and Age, $F(1,56)=11.75, p=.001, \eta_{G}^{2}=$ .03 (see Figure 3).

This three-way interaction was investigated by conducting two separate two-way ANOVAs (one for each Age group) with the factors Sequence and Response. For the older adults, there was no main effect of Sequence, $F(1,28)=3.40, p=.08, \eta_{G}^{2}=.01$, no main effect of Response, $F(1,28)=0.43, p=.52, \eta_{G}^{2}=.002$, and no two-way interaction, $F(1,28)$ $<0.01, p=.93, \eta_{G}^{2}=.009$. For the younger adults, there was no main effect of Sequence, $F(1,28)=2.16, p=.15, \eta_{G}^{2}=.01$, no main effect of Response, $F(1,28)=0.32, p=.58$, $\eta_{G}^{2}=.001$, but the two-way interaction was significant, $F(1,28)=16.08, p<.001, \eta_{G}^{2}=$ 
Table 2

Bayes factors for various models predicting $n-2$ task repetition costs as the dependent variable; separate analyses were conducted for response times (RTs), $\log (R T)$, and accuracy. Note that all models also included the random effects factor of Subject. All reported Bayes factors are against a denominator of predicting response time from just the random effects factor of Subject. "+" refers to the addition of a main effect, and "*" refers to an interaction between factors

\begin{tabular}{llr}
\hline DV & Model & BF \\
\hline & & \\
RT & Response (R) & 541.00 \\
& Age (A) & 0.25 \\
& $\mathrm{R}+\mathrm{A}$ & 142.00 \\
& $\mathrm{R}+\mathrm{A}+\mathrm{R} * \mathrm{~A}$ & 37.10 \\
& & \\
\hline \multirow{2}{*}{$\log (\mathrm{RT})$} & $\mathrm{R}$ & \\
& $\mathrm{A}$ & 513.00 \\
& $\mathrm{R}+\mathrm{A}$ & 0.34 \\
& $\mathrm{R}+\mathrm{A}+\mathrm{R} * \mathrm{~A}$ & 759.00 \\
& & \\
\hline \multirow{4}{*}{ Accuracy } & $\mathrm{R}$ & \\
& $\mathrm{A}$ & 40.50 \\
& $\mathrm{R}+\mathrm{A}$ & 0.22 \\
& $\mathrm{R}+\mathrm{A}+\mathrm{R} * \mathrm{~A}$ & 1032.00 \\
\hline
\end{tabular}

.08. The $\mathrm{n}-2$ task repetition cost (accuracy ABA minus accuracy CBA) was $-0.025[95 \%$ $\mathrm{CI}=-0.036,-0.014]$ for $\mathrm{n}-2$ response switches $[t(30)=-4.57, p<.001$, Cohen's $d=-0.848]$, but was $0.013[-0.001,0.027]$ for $\mathrm{n}-2$ response repetitions $[t(30)=1.83, p=.08$, Cohen's $d$ $=0.341]$. That is, there was an $\mathrm{n}-2$ task repetition cost for $\mathrm{n}-2$ response switches, but a (non-significant) n-2 task repetition benefit for $\mathrm{n}-2$ response repetitions.

Bayesian Analysis. For the accuracy analysis (see lower panel of Table 2), the full main effects plus interaction model was the overall best model with a BF of 1032; the next-best model was the main effect of Response with a $\mathrm{BF}$ of 40.50 . The $\mathrm{BF}$ in favour of the full main effects plus interaction model compared to the next-best model is 1032/40.50 $=25.48$, which provides "strong" evidence in favour of the full interaction model.

\section{General Discussion}

A prominent hypothesis for age-related decline in cognitive function is the inhibition deficit hypothesis (Hasher et al., 2007; Hasher \& Zacks, 1988; Hasher et al., 1999), which suggests that inhibition becomes less efficient as we age. Task switching designs have been used to assess age-related decline in cognitive inhibition - as measured by the n-2 task repetition cost - and find no clear evidence for age-related decline (Lawo et al., 2012; Mayr, 2001; Pettigrew \& Martin, 2016; Rey-Mermet et al., in press; Schuch, 2016). 
The purpose of the current study was to assess measures of the n-2 task repetition cost in older adults in comparison to younger adults whilst controlling for episodic retrieval, which has recently been shown to contaminate measures of the n-2 task repetition cost (Grange et al., 2017). Thus, we argue that controlling for this provides a better measure of any putative inhibitory mechanism in task switching.

Consistent with our earlier work, we found reduced $n-2$ task repetition costs for episodic matches ( $\mathrm{n}-2$ response repetitions) compared to episodic mismatches ( $\mathrm{n}-2$ response switches). Thus, when episodic retrieval is controlled, estimates of inhibition are reduced. Importantly for the current paper, this pattern was equivalent in the older adults and younger adults for response times, which provides no evidence for an inhibitory deficit in older adults.

The reduction of n-2 task repetition costs for episodic matches was mirrored by the accuracy data for younger adults, but older adults showed no clear effects in the accuracy data. Whilst older adults showed no effects of Task Sequence or Response Repetition on performance, younger adults showed n-2 repetition costs for response switches, and a (nonsignificant) n-2 task repetition benefit for response repetitions. That is, there were episodic retrieval contributions to the accuracy data for younger adults, but not older adults. This difference in performance accuracy between older and younger adults is hard to interpret because older adults' accuracy was near ceiling. However, it could point to there being age-related differences in episodic retrieval and inhibition manifesting in accuracy data.

\section{Aging \& Task Switching}

These findings help paint a clearer picture of there being no age-related deficit of inhibition in task switching (cf., Hasher et al., 2007, 1999). Evidence to date has been mixed, with Mayr (2001) and Pettigrew and Martin (2016) finding larger n-2 task repetition costs for older adults than younger adults, and with Lawo et al. (2012), Schuch (2016), and ReyMermet et al. (in press) finding no age-related difference of the $\mathrm{n}-2$ task repetition cost at the behvioural level. Although all studies used different methodologies, an examination of these reveal no clear reason why this would cause the varying outcomes. For example, all studies were cued task switching designs and required some form of stimulus classification with multivalent response requirements. However, all studies include the confound of episodic retrieval, so it is not clear whether the estimates of $n-2$ task repetition cost reflect inhibition, episodic retrieval, or both. The contribution of episodic retrieval to the $\mathrm{n}-2$ task repetition cost appears strong and robust (see the current experiment and the three experiments reported by Grange et al., 2017), so it was important to revisit this topic and control for episodic retrieval effects.

These findings are consistent with recent meta-analyses which demonstrate no clear age-related effect on other key measures of task switching performance. For example, Wasylyshyn et al. (2011) found no age-related decline in switch costs (the cost associated with switching tasks compared to repeating tasks), which has been a prominent measure of "cognitive control" in the task switching literature (see Grange \& Houghton, 2014; Kiesel et al., 2010; Logan, 2003; Vandierendonck et al., 2010, for reviews and controversy surrounding this measure). More recently, Grange and Becker (in press) found no evidence for agerelated decline in the response congruency effect (impaired performance when a stimulus is associated with multiple responses dependent upon task, in comparison to a stimulus which 
is associated with a single response regardless of task). As these costs have been associated with cognitive control processes, the findings are consistent with the view that age-related decline of cognitive control is not as ubiquitous as previously thought (Verhaeghen, 2011).

\section{Aging \& Inhibition}

The current findings also sit within a wider literature which is beginning to question whether the inhibitory deficit hypothesis of age-related decline in cognition is correct. During the final preparation of this manuscript, we became aware of a large-scale study examining individual- and age-differences in inhibition (Rey-Mermet et al., in press). The study presented 130 younger and 159 older adults with a battery of 11 cognitive tasks thought to measure cognitive inhibition, including the $n-2$ task repetition cost. The researchers found no age-related difference in n-2 task repetition cost. Due to the battery of tasks presented, the researchers were also able to model inhibition at the latent variable level, and then to explore age-related differences at this latent level. Two factors of inhibition were found - inhibition of pre-potent responses and resistance to distractor interferencebut no clear general age-related difference was present. Although the latent model showed impairment in pre-potent response inhibition in older adults, it showed better performance in older adults for distractor-interference resistance. Overall, though, this latent model had little explanatory power, which calls into question both inhibition as a general construct, and any age-related decline in it.

Our findings are in line with this latter conclusion, finding no age-related difference in inhibition in task switching when controlling for an important confound.

\section{References}

Bates, D., Mächler, M., Bolker, B., \& Walker, S. (2015). Fitting linear mixed-effects models using lme4. Journal of Statistical Software, 67. doi: 10.18637/jss.v067.i01

Borson, S., Scanlan, J., Brush, M., Vitaliano, P., \& Dokmak, A. (2000). The Mini-Cog: a cognitive vital signs measure for dementia screening in multi-lingual elderly. International Journal of Geriatric Psychiatry, 15, 1021-1027. doi: 10.1002/1099-1166(200011)15:11<1021::AIDGPS234>3.0.CO;2-6

Friedman, N. P., \& Miyake, A. (2004). The relations among inhibition and interference control functions: A latent variable analysis. Journal of Experimental Psychology: General, 133, 101-135. doi: 10.1037/00963445.133.1.101

Gamboz, N., Russo, R., \& Fox, E. (2002). Age differences and the identity negative priming effect: An updated meta-analysis. Psychology and Aging, 17, 525-531. doi: 10.1037/0882-7974.17.3.525

Gorfein, D. S., \& Brown, V. R. (2007). Saying no to inhibition. In D. S. Gorfein \& C. M. MacLeod (Eds.), Inhibition in cognition (pp. 103-124). Washington, DC: Americal Psychological Association.

Gorfein, D. S., \& MacLeod, C. M. (Eds.). (2007). Inhibition in cognition. Washington, DC: Americal Psychological Association.

Grange, J. A., \& Becker, R. B. (in press). The effect of aging on response congruency in task switching: A meta-analysis. Journals of Gerontology Series B: Psychological Sciences and Social Sciences.

Grange, J. A., \& Houghton, G. (Eds.). (2014). Task switching and cognitive control. New York, NY: Oxford University Press. 
Grange, J. A., Kowalczyk, A. W., \& O'Loughlin, R. (2017). The effect of episodic retrieval on inhibition in task switching. Journal of Experimental Psychology: Human Perception and Performance, 43, 1568-1583. doi: 10.1037/xhp0000411

Hasher, L., Lustig, C., \& Zacks, R. T. (2007). Inhibitory mechanisms and the control of attention. In A. Conway, C. Jarrold, M. Kane, A. Miyake, \& J. Towse (Eds.), Variation in working memory (pp. 227-249). New York, NY: Oxford University Press.

Hasher, L., \& Zacks, R. T. (1988). Working memory, comprehension, and aging: A review and a new view. In G. H. Bower (Ed.), The psychology of learning and motivation (Vol. 2, pp. 193-225). San Diego, CA: Academic Press. doi: 10.1016/S0079-7421(08)60041-9

Hasher, L., Zacks, R. T., \& May, C. P. (1999). Inhibitory control, circadian arousal, and age. In D. Gopher \& A. Koriat (Eds.), Attention and Performance XVII. Cognitive regulation of performance: Interaction of theory and application (pp. 653-675). Cambridge, MA: MIT Press.

Hommel, B. (2004). Event files: feature binding in and across perception and action. Trends in Cognitive Sciences, 8, 494-500. doi: 10.1016/j.tics.2004.08.007

Kiesel, A., Steinhauser, M., Wendt, M., Falkstein, M., Jost, K., Philipp, A., \& Koch, I. (2010). Control and interference in task switching. Psychological Bulletin, 136, 849-874. doi: $10.1037 / \mathrm{a} 0019842$

Koch, I., Gade, M., Schuch, S., \& Philipp, A. M. (2010). The role of inhibition in task switchinng: A review. Psychonomic Bulletin \& Review, 17, 1-14. doi: 10.3758/PBR.17.1.1

Kramer, A. F., Humphrey, D. G., Larish, J. F., \& Strayer, D. L. (1995). Aging and inhibition: Beyond a unitary view of inhibitory processing in attention. Psychology and Aging, 9, 491512. doi: 10.1037/0882-7974.9.4.491

Lawo, V., Philipp, A. M., Schuch, S., \& Koch, I. (2012). The role of task preparation and task inhibition in age-related task-switching deficits. Psychology and Aging, 27, 1130-1137. doi: $10.1037 / \mathrm{a} 0027455$

Logan, G. D. (1988). Toward an instance theory of automatization. Psychological Review, 95, 492-527. doi: doi:10.1037/0033-295X.95.4.492

Logan, G. D. (2003). Executive control of thought and action: In search of the wild homunculus. Current Directions in Psychological Science, 12, 45-48. doi: https://doi.org/10.1111/14678721.01223

MacLeod, C. M. (2007). The concept of inhibition in cognition. In D. S. Gorfein \& C. M. MacLeod (Eds.), Inhibition in cognition (pp. 3-23). Washington, DC: Americal Psychological Association.

MacLeod, C. M., Dodd, M. D., Sheard, E. D., Wilson, D. E., \& Bibi, U. (2003). In opposition to inhibition. Psychology of Learning and Motivation, 43, 163-214.

Mayr, U. (2001). Age differences in the selection of mental sets: The role of inhibition, stimulus ambiguity, and response-set overlap. Psychology and Aging, 16, 96-109. doi: 10.1037/08827974.16.1.96

Mayr, U. (2002). Inhibition of action rules. Psychonomic Bulletin E Review, 9, 93-99. doi: 10.3758/BF03196261

Mayr, U., \& Keele, S. W. (2000). Changing internal constraints on action: The role of backward inhibition. Journal of Experimental Psychology: General, 129, 4-26. doi: http://dx.doi.org/10.1037/0096-3445.129.1.4

Morey, R. D., \& Rouder, J. N. (2015). BayesFactor: Computation of Bayes factors for common designs [Computer software manual]. Retrieved from http: //CRAN.R-project.org/package=BayesFactor (R package version 0.9.12-2)

Nigg, J. T. (2000). On inhibition/disinhibition in developmental psychopathology: Views from cognitive and personality psychology and a working inhibition taxonomy. Psychological Bulletin, 126, 200-246. doi: 10.1037/0033-2909.126.2.220

Pettigrew, C., \& Martin, R. C. (2016). The role of working memory capacity and interference 
resolution mechanisms in task switching. Quarterly Journal of Experimental Psychology, 69, 2431-2451. doi: 10.1080/17470218.2015.1121282

Philipp, A. M., \& Koch, I. (2006). Task inhibition and task repetition in task switching. European Journal of Cognitive Psychology, 18, 624-639. doi: 10.1080/09541440500423269

Pierce, J. W. (2007). PsychoPy-Psychophysics software in Python. Journal of Neuroscience Methods, 162, 8-13. doi: https://doi.org/10.1016/j.jneumeth.2006.11.017

R Core Team. (2015). R: A language and environment for statistical computing [Computer software manual]. Vienna, Austria. Retrieved from http://www.R-project.org/

Rey-Mermet, A., Gade, M., \& Oberauer, K. (in press). Should we stop thinking about inhibition? searching for individual and age differences in inhibition ability. Journal of Experimental Psychology: Learning, Memory \& Cognition. doi: 10.1037/xlm0000450

Rouder, J. N., Morey, R. D., Verhagen, J., Swagman, A. R., \& Wagenmakers, E.-J. (2017). Bayesian analysis of factorial designs. Psychological Methods, 22, 304-321. doi: 10.1037/met0000057

Schuch, S. (2016). Task inhibition and response inhibition in older vs. younger adults: A diffusion model analysis. Frontiers in Cognition, 7, 1722. doi: https://doi.org/10.3389/fpsyg.2016.01722

Spapé, M. M., \& Hommel, B. (2014). Sequential modulations of the Simon effect depend on episodic retrieval. Frontiers in Psychology, 5. doi: 10.3389/fpsyg.2014.00855

Vandierendonck, A., Liefooghe, B., \& Verbruggen, F. (2010). Task switching: Interplay of reconfiguration and interference. Psychological Bulletin, 136, 601-626. doi: 10.1037/a0019791

Verhaeghen, P. (2011). Aging and executive control: Reports of a demise greatly exaggerated. Current Directions in Psychological Science, 20, 174-180. doi: 10.1177/0963721411408772

Verhaeghen, P., \& De Meersman, L. (1998). Aging and the Stroop effect: A meta-analysis. Psychology and Aging, 13, 120-126. doi: 10.1037/0882-7974.13.1.120

Wasylyshyn, C., Verhaeghen, P., \& Sliwinski, M. J. (2011). Aging and task switching: A metaanalysis. Psychology and Aging, 26, 15-20. doi: 10.1037/a0020912

Yesavage, J. A., Brink, T. L., \& Rose, T. L. (1983). Development and validation of a geriatric depression screening scale: A preliminary report. Journal of Psychiatric Research, 17, 37-49. doi: $10.1016 / 0022-3956(82) 90033-4$ 